\title{
Mémoire du futur, from old roots to new shoots. Patrick Geddes in India (1914-1924)
}

\author{
Adrián Torres Astaburuaga \\ Éva Chaudier \\ Nicolas Tixier
}

\section{GEDDES IN INDIA}

Botaniste et biologiste écossais, Patrick Geddes développe une pensée et une pratique du projet urbain qui participe d'un mouvement que l'on pourrait rétrospectivement qualifier de proto-écologiste, en posant que l'écologie n'est pas seulement une question environnementale, mais bien aussi une question

Adrián Torres Astaburuaga, architecte diplômé de l'École technique supérieure d'architecture de Valencia, activiste urbain et chercheur à l'École d'architecture de Barcelone et à l'École supérieure d'art de l'agglomération d'Annecy, membre du collectif Solar Corona.

adtoras@gmail.com

Éva Chaudier, designer, diplôme supérieur d'arts appliqués, mention design d'espace (lycée La Martinière-Diderot, Lyon) et master Urbanisme et aménagement, parcours design urbainprojet de fin d'études, mention recherche (Institut d'urbanisme de Grenoble et École nationale supérieure d'architecture de Grenoble).

eva.chaudier@virginbox.fr

Nicolas Tixier, professeur à l'École nationale supérieure d'architecture de Grenoble et à l'École supérieure d'art de l'agglomération d'Annecy, chercheur au Cresson, UMR Ambiances, architectures, urbanités, membre du collectif BazarUrbain.

nicolas.tixier@grenoble.archi.fr 
sociale et économique ${ }^{1}$. Ses méthodes interdisciplinaires placent l'homme en relation avec un large écosystème à considérer, dont le contexte urbain fait pleinement partie. Sa pensée, relativement oubliée par le récit des modernes, appréhende le développement urbain à partir des sciences naturelles et des sciences sociales, en dépassant leur distinction et en affichant une position très critique envers ce qui relève, selon lui, d'un ordre paléotechnique, à savoir une croissance urbaine incontrôlée ou encore l'industrialisation sauvage sans ménagement aucun envers l'environnement ou ceux qu'elle emploie, et qui de plus est à la base de l'épuisement de ressources telles que le charbon. Il critique un système socio-économique où la dissipation de l'énergie sert uniquement le profit de quelques-uns. Il annonce alors le passage de cet ordre paléotechnique à un ordre néotechnique prônant la conservation de l'énergie et l'organisation d'un environnement orientées vers une évolution sociétale possible tant pour la collectivité que pour l'individu. Dans ses études, Geddes fait un lien direct entre la qualité de l'ambiance urbaine et le progrès social. La compréhension et l'articulation des différents paramètres qui font la société urbaine lui permettent de pointer en particulier la période entre la fin du $\mathrm{XIX}^{\mathrm{e}}$ siècle et le début du $\mathrm{XX}^{\mathrm{e}}$ siècle durant laquelle l'industrialisation altère gravement les conditions de vie.

Aujourd'hui, l'écologie a quitté les secteurs alternatifs et minoritaires pour se retrouver au cœur des discours des institutions publiques autant que privées, mais aussi des urbanistes, au risque, nous le savons tous, de la superficialité voire du marketing. Les idées, les études et les pratiques urbaines de Geddes, développées il y a maintenant cent ans, nous semblent tout à fait pertinentes pour comprendre l'origine de ce proto-écologisme ou de la planification organique (Oyón, 2011).

À la différence des urbanistes actuels qui développent une posture écologique, Geddes n'avait pas une notion claire de l'épuisement des ressources

1. Cet article s'appuie sur trois travaux de recherche menés conjointement :

Adrián Torres Astaburuaga, Valencia, ville en mutation: mémoire naturelle, stratigraphie urbaine, réactivation par l'usage, doctorat ETSAB-UPC (École supérieure d'architecture de Barcelone), José Luis Oyón, Manuel Guardia, Nicolas Tixier (sous la dir. de), 2013-2017 et un DSRA à l'École supérieure d'art de l'agglomération d'Annecy - programme ECHOS en collaboration avec le MAMCO, 2014-2016.

Naïm Aït-Sidhoum (sous la dir. de), Nicolas Tixier, Adrián Torres et al., Bangalore : pour l'invention d'un tiers patrimoine, programme de recherche MCC « Pratiques interculturelles dans les processus de patrimonialisation », École supérieure d'art de l'agglomération d'Annecy (sous la dir. de), Srishti Institute of Art de Bangalore, Cresson - UMR AAU - ENSA Grenoble, 2014-2016. Éva Chaudier, From Old Roots to New Shoots, entre patrimoine et projet, relecture contemporaine de Patrick Geddes, master recherche en design urbain, Nicolas Tixier (sous la dir. de), Institut d'urbanisme de Grenoble / Cresson - UMR AAU - ENSA Grenoble, 2015. 
naturelles ni même des conflits pouvant en découler. La crise du pétrole, le changement climatique, la biodiversité menacée n'étaient pas d'actualité à l'époque. Formé à la biologie puis à la géographie environnementaliste, Geddes avait une vision fondée sur une forte sensibilité envers la nature. Ses ouvrages montrent, souvent de façon implicite, les limites à ne pas dépasser concernant, par exemple, la contamination des aquifères et des rivières, la quantité des effluents industriels et leurs conséquences sur la santé publique.

Patrick Geddes, comme ses référents les plus proches, à savoir les romantiques révolutionnaires tels que John Ruskin, Thomas Carlyle, William Morris, ou les géographes anarchistes tels que Pierre Kropotkin et Élisée Reclus, avaient la conviction de l'union ou d'une continuité entre l'homme et la nature. Son approche n'était pas pour autant protectionniste ; il avait plutôt la volonté de prendre pleinement en compte le lien entre l'homme et la nature dans son étude des villes et de leurs territoires.

Mais Patrick Geddes, à la différence d'auteurs comme Henry David Thoreau, qui était plutôt un détracteur du milieu urbain et de la civilisation de son époque, a orienté son travail vers une renaturalisation de la cité et de la culture. En cela, Geddes a fortement influencé l'urbaniste et théoricien américain Lewis Mumford, qui a écrit l'introduction de Patrick Geddes in India, un ouvrage à la base du travail d'action et de recherche décrit dans cet article.

En novembre 1914, Patrick Geddes est appelé en Inde pour réaliser différents reports en tant que planning consultant. Invité par Lord Pentland, gouverneur de la région indienne de Mantras et ancien ami, il part vers l'Inde avec son fils Alasdair Geddes, emportant l'exposition «Cities Exhibition », qui représente pour lui des années de recherches et de collectes. Un bateau allemand coule le Clan Grant, navire sur lequel se trouve toute la documentation de l'exposition, la perdant en totalité. Geddes ira et retournera en Inde pour y rester finalement durant la guerre, jusqu'en 1924. Mais cette guerre tuera son fils Alasdair, recruté par l'armée britannique, mort sur le front français, précédant de peu son épouse, décédée en 1917 d'une maladie tropicale transmise par un moustique pendant leur séjour en Inde. Ces terribles événements sont très significatifs pour comprendre l'état d'esprit de Patrick Geddes lorsqu'il développe ses travaux en Inde.

En Inde coloniale, Geddes réalise entre 1914 et 1924 des études que l'on pourrait qualifier aujourd'hui de « diagnostics prospectifs » sur plusieurs villes et villages du pays comme Indore, Balrampur, Madurai, Calcutta, etc. Ses exposés, ses préconisations et sa méthode furent à cette époque en avance sur son temps et peu compris. La volonté de modernisation et d'une certaine manière d'occidentalisation que les gouverneurs de l'Inde anglaise avaient se trouvait en opposition avec les analyses et les propositions qu'il faisait. Peu mis en avant pendant de longues années, ces travaux sont revisités aujourd'hui par de nombreux champs disciplinaires (urbanisme, géographie, paysage, 
éducation, etc.). En effet, les points de vue de Geddes sont à la base de positionnements écologistes et postcoloniaux contemporains, en plaçant l'homme, avec ses savoir-faire, sa culture locale, sa recherche du bien-être, dans un contexte écosystémique qui se retrouve au cœur de sa logique de planification urbaine.

Les études indiennes réalisées sur une cinquantaine de villes ou villages se composent d'un nombre de volumes d'ampleur variable selon le cas. Le report de la ville d'Indore reste le plus développé (Geddes, 1918) - et c'est sur celui-ci que nous nous sommes le plus appuyés. Une sélection d'études et de propositions urbaines menées à terme entre les années 1915 et 1919 ont été éditées en 1947 par Jaqueline Tyrwhitt, en collaboration avec Henry Vaughan Lanchester, Arthur Geddes, le fils de Patrick Geddes, ainsi qu'Anthony Denney pour le travail photographique. L'ouvrage, intitulé Patrick Geddes in India, est composé d'une sélection raisonnée parmi les différents reports indiens réalisés (Tyrwhitt, 1947). Très peu connu dans les pays non-anglophones, il n'a été ni réédité ni traduit (figure $1^{2}$ ). À notre connaissance, un seul ouvrage important porte sur les travaux de Geddes en Inde. Il s'agit d'une monographie remarquable publiée en italien par Giovanni Ferraro en 1998 (Ferraro, 1998).

L'approche de la ville, le processus méthodologique que Patrick Geddes met en place en articulant relevés, diagnostics et propositions - souvent au sein d'un même mouvement -, sont aujourd'hui d'une actualité et sans doute d'une validité étonnantes. Dans sa préface de Patrick Geddes in India, Lewis Mumford met en évidence la grande portée, selon lui, des propositions faites par Geddes, le présentant comme une figure importante de par ses postures, sa méthodologie, ses éléments de théorie, mais aussi la façon dont il esquisse le projet. Mumford remarque parmi les premiers l'intérêt du survey before planning :

«Today no competent planner would think of putting forward a comprehensive scheme for city improvement without a preliminary survey of the geology, the geography, the climate, the economic life, and the social institutions of the city and its region. But this commonplace of technique is the direct result of Geddes's advocacy of the civic survey as indispensable to planning: "Diagnosis before treatment." » (Mumford dans Tyrwhitt, 1947, p. 8)

Cette méthode, mise en pratique en Inde, développe les éléments d'analyse urbaine et de projet élaborés et énoncés pour d'autres lieux, en particulier la ville et la région d'Édimbourg. Elle se compose de deux moments : le survey before planning et le conservative surgery.

2. Nous tenons à remercier pour leur aimable autorisation de reproduction les éditions Lund Humphries à Londres. 


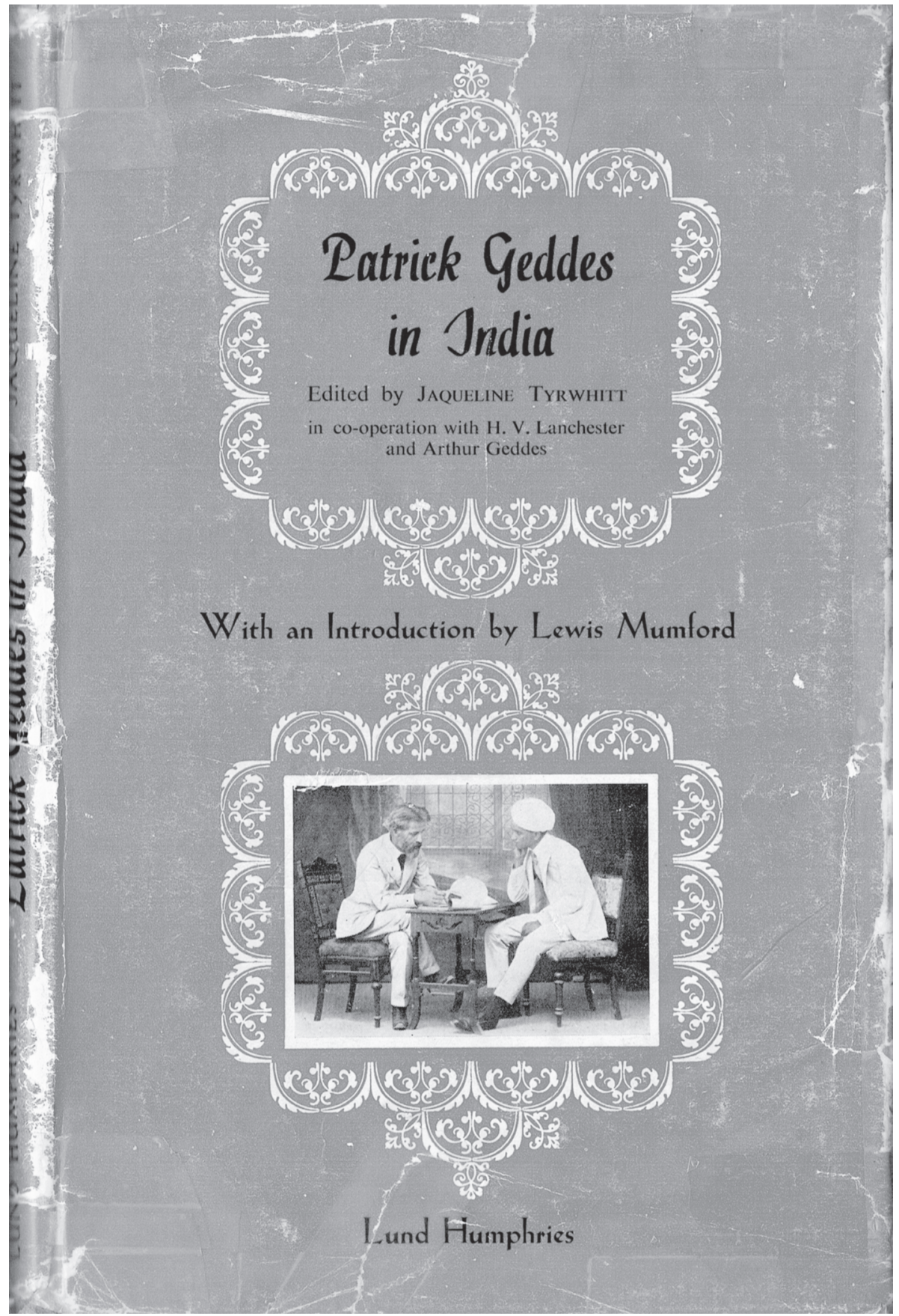

Figure 1 - Couverture de l'édition : GedDes, P. Patrick Geddes in India, édité par J. Tyrwhitt, H. V. Lanchester et A. Geddes, introduction de Lewis Mumford, Londres, Lund Humphries, 1947. 
Le survey before planning consiste en une étude assez rigoureuse fondée sur une cartographie du vivant, des mentalités et de la société que Geddes relie aux caractéristiques d'espace, de temps et de fonction. Il pointe des situations concrètes du moment analysé, sans oublier la vision historique ni l'interprétation et la valorisation d'éléments présents pour construire une vision du futur. En cela, Geddes propose une analyse sociologique autant qu'anthropologique et géographique. Pour développer cette analyse dans tous ses reports indiens, il fait allusion à la marche comme façon de pénétrer intimement les labyrinthes de la ville : starting by walking (Geddes, 1915, p. 53), active peregrination (Geddes, 1917a, p. 1), repeated perambulation (Geddes, 1917b, p. 1 ; Geddes, 1918, p. 1). Pour Geddes, la marche est l'outil essentiel de connaissance d'un territoire et de ses habitants. Pour Madras, il explique que le processus de planification est une conséquence directe du travail d'arpenteur :

«The work cannot be done in the office with ruler and parallels, for the plan must be sketched out on the spot, after wearying hours of perambulationcommonly amid sights and odours wich neither Brahmin nor Briton has generally schooled himself to endure. » (Geddes dans Tyrwhitt, 1947, p. 44) ${ }^{3}$

La référence à la marche comme base du survey pour le développement des quartiers et de la ville sera présente dans tous les reports indiens.

Le conservative surgery, littéralement " chirurgie conservatrice » ou de façon plus contemporaine «microchirurgie urbaine », est proposé pour intervenir dans les quartiers informels de différentes cités. Pour ces quartiers en apparence chaotique, Geddes, contrairement aux postulats modernistes, propose des interventions délicates, minutieuses et pensées de manière à limiter les démolitions et les ouvertures trop excessivement hygiénistes et fonctionnalistes (figures 2 et 2 bis $^{4}$ ). Il travaille à respecter l'existant, le déjà-là, en économisant de fait sur les dépenses tant énergétiques que budgétaires. Il met en valeur des petits espaces naturels en ville ou encore, par exemple, souligne l'importance d'avoir des arbres fruitiers productifs comme source d'autonomie alimentaire. Mais, tout autant que l'aspect botanique, les aspects locaux des savoir-faire, des traditions et des cultes religieux sont repérés, analysés puis valorisés en tant qu'éléments structurant de la cité. Par exemple, les petits espaces publics reliés au temple, parfois associés à un arbre sacré ou une source d'eau, sont identifiés comme des espaces symboliques d'importance dans la construction d'un réseau de petites places qui constituent autant d'endroits pour de potentielles petites

3. «Le travail ne peut pas être fait dans un bureau avec la règle et l'équerre, le plan doit être puisé sur le site, après avoir fait des heures de marche - souvent au milieu de situations et d'odeurs que ni le Brahmane ni le Britannique ne sont habitués à supporter. »

4. Nous tenons à remercier pour leur aimable autorisation de reproduction les éditions Lund Humphries à Londres. 

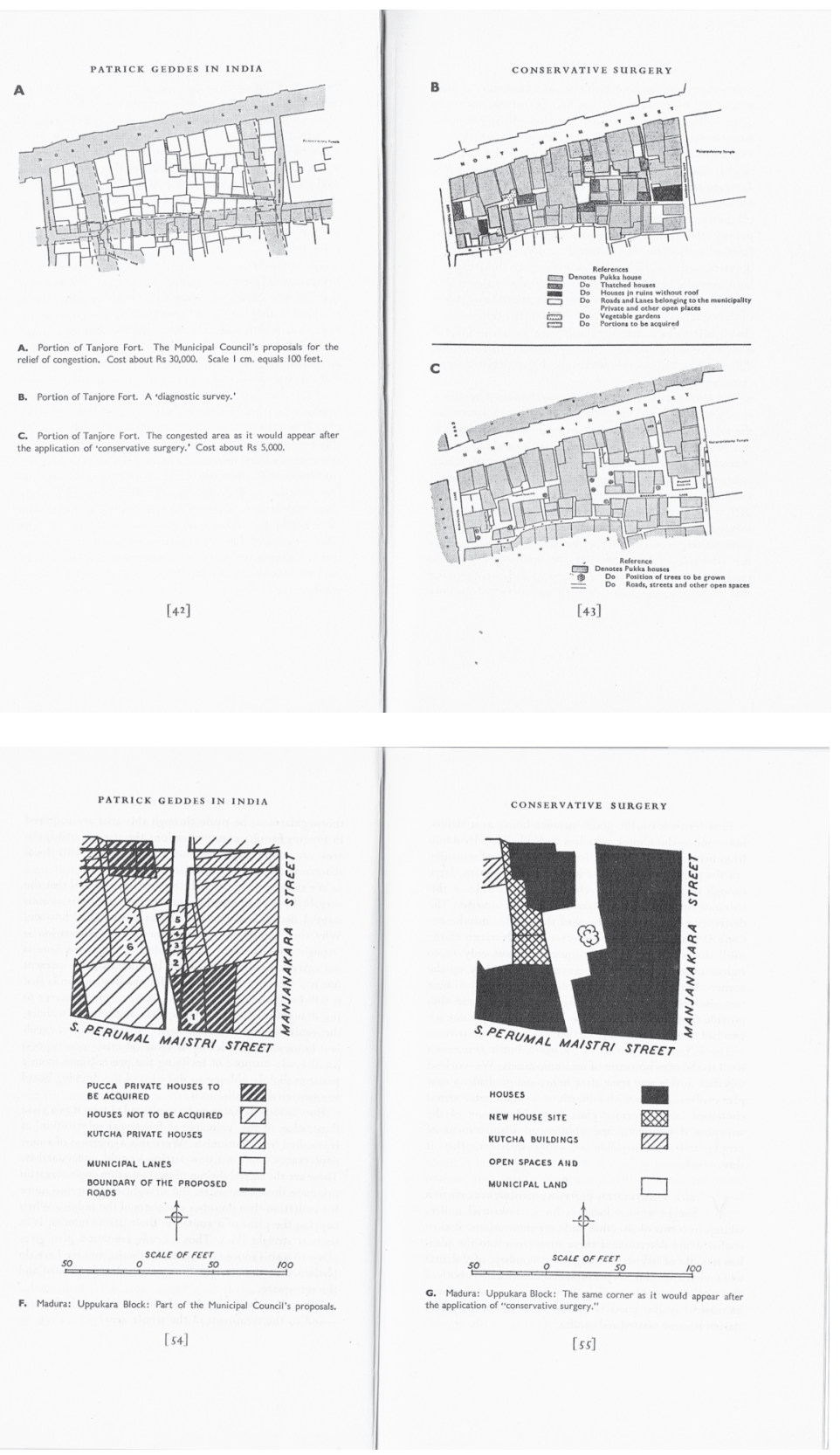

Figure 2 et 2 bis - Conservative Surgery in Madura et Tanjore Fort (noms de figures) présentés dans l'édition : GEDDES, P. Patrick Geddes in India, édité par J. Tyrwhitt, H. V. Lanchester et A. Geddes, introduction de Lewis Mumford, Londres, Lund Humphries, 1947. 
ouvertures (figure $3^{5}$ ). En particulier dans l'ouvrage Temple Cities (1919), Geddes interprète l'architecture et l'urbanisme indien selon des critères nonethnocentriques, en mettant en lumière l'importance des différentes religions indiennes dans la configuration des villes.

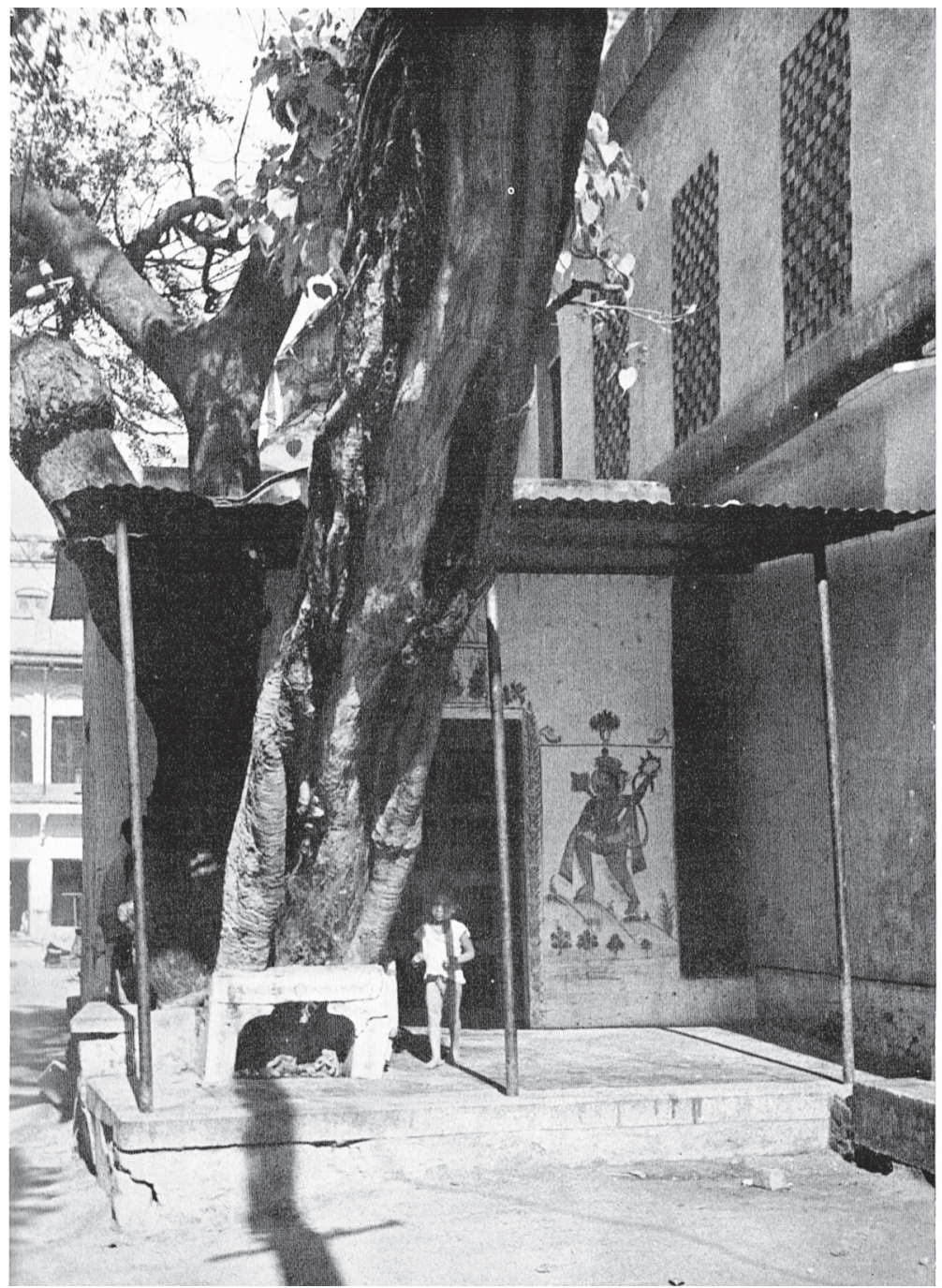

Figure 3 - Photographie de Anthony Denney présente dans l'édition : GedDEs, P. Patrick Geddes in India, édité par J. Tyrwhitt, H. V. Lanchester et A. Geddes, introduction de Lewis Mumford, Londres, Lund Humphries, 1947.

5. Nous tenons à remercier pour leur aimable autorisation de reproduction les éditions Lund Humphries à Londres et Celia Denney. 
Ces analyses concernent également les questions hydrauliques qui sont d'une importance vitale dans ses reports. En effet, la distribution de l'eau, la valorisation des canaux d'irrigation et d'assainissement ou nalas, la mise en valeur des lacs et des marécages représentent pour Geddes des éléments essentiels de ressources en eau pour une ville et ses habitants, que ce soit en tant que système écologique de drainage ou comme moyen de maintenir un niveau phréatique sain.

Dans une époque coloniale où l'occidentalisation culturelle était de mise, Geddes pense, d'une façon qui pourrait être interprétée comme pré-postcoloniale, la microéconomie locale, l'importance et les rôles des métiers manuels, le respect des religions et des lieux de culte, vertèbres des quartiers. Pour Geddes, la culture se transmet à travers l'apprentissage social et par l'oralité. C'est pourquoi les femmes indiennes jouent un rôle si important à ses yeux, celui de dépositaires et de véhicules de la mémoire de ce tissage du quotidien. Ce social heritage est l'une des notions indispensables pour comprendre l'évolution de la pensée geddesienne, et notamment l'importance qu'il donne aux questions d'éducation et de partage des connaissances (Ferraro, 1998).

Il est passionnant aussi de noter dans ses reports indiens sa vision avantgardiste du rôle des techniciens des différents services urbains et de la pertinence de la coopération entre les acteurs impliqués dans l'organisation d'une ville. Geddes parle d'un technicien qualifié qui rendrait service à la société et dont les connaissances et la compréhension des caractéristiques locales ainsi que le contact avec les habitants seraient essentiels. Il imagine un technicien ayant une appétence sociale et un enthousiasme civique lui permettant de parcourir les quartiers et d'encourager les habitants à participer à l'amélioration de l'ambiance commune :

«Local knowledge and understanding are essential to the town planner, together with consideration and tact when dealing with the individual requirements of citizens. Other requirements are powers of social appeal and civic enthusiasm that will enable him to arouse neighbourhood after neighbourhood to participate in schemes of improvements instead of remaining indifferent to them. Only in this way can he gradually inspire the city as a whole. For such work, moral influence and energy are needed in no small measure. [...] Above all the planner must possess a sustained courage and a contagious glow of feeling. » (Geddes dans Tyrwhitt, 1947, p. 37) ${ }^{6}$

6. « La connaissance et la compréhension locale sont essentielles pour le town planner ainsi que la considération et le tact lorsqu'il traite les exigences individuelles des citoyens. Les autres exigences sont les pouvoirs du social appeal et de l'enthousiasme civique qui lui permettront de susciter quartier par quartier la participation aux plans d'amélioration, au lieu de l'indifférence. C'est seulement de cette manière qu'il peut progressivement inspirer la cité comme un tout. Pour cela, influence morale et énergie sont nécessaires en grande mesure. [...] Surtout le planner doit posséder un courage soutenu et partager avec ardeur son ressenti. » 
Chez Geddes, le planning n'est pas seulement un instrument d'amélioration des conditions environnementales pour la vie de chacun, il est également un outil de décision collective permettant de collecter et débattre des éléments du passé et du présent, autant pour les préserver que pour les transmettre. Autrement dit se pose la question de savoir quelles empreintes conserver et mettre en perspective dans le projet pour le tourner vers l'avenir.

Geddes insiste sur le fait qu'un technicien doit être doué d'une générosité basée sur l'écoute et d'une capacité à apprendre et à valoriser les connaissances aussi bien de l'érudit que du vagabond. Ces réflexions seront développées bien plus tard, en particulier par Michel de Certeau.

Geddes énonce de façon très avant-gardiste le besoin de coordination locale entre les différents agents et les différentes municipalités :

«But it is plainly time for the co-operation of the regional geographer with the hygienist, and of both with the concrete sociologist, the student of country and town, of village and city; and also for the furtherance of their labours, the discussion of them in detail, in friendly conferences representative of all the various groupings and interests concerned. »(Geddes, 1915, p. 44) ${ }^{7}$

Pour synthétiser l'approche de Geddes quant à la finalité de tout projet urbain, nous citerons ce qu'il écrit sur Madura :

" Town Planning is not mere Place-Planning, nor even work-planning. If it is to be successful, it must be folk-planning. This means that its task is not to coerce people into new places against their associations, wishes, and interest. [...] Instead its task is to find the right places for each sort of people; places where they will really flourish. » (Geddes dans Tyrwhitt, 1947, p. 22)

\section{ENTRE PATRIMOINE ET PROJET}

Les nombreuses pièces écrites, graphiques et cartographiques produites au cours de ses séjours en Inde montrent à quel point sa perception du déjà-là est écosystémique, plus qu'organique. Sa démarche, passionnante, dévoile des allersretours permanents entre microsystème et macrosystème, pour comprendre la vie urbaine et humaine qui s'y développe et l'accompagner par le projet.

Par l'articulation des aspects techniques, humains ou encore économiques, il s'agit pour Geddes de ne jamais perdre de vue la relation vitale qui lie l'homme à son environnement et de l'envisager comme un tout. Sa réflexion est globale alors que l'action et les interventions prônées se déclinent et s'établissent au cas par cas. Ainsi les nombreuses cartes redessinées par Geddes alternent le détail de l'aménagement urbain et l'esquisse de l'organisation territoriale.

7. «C'est le moment de la coopération entre le géographe régional et l'hygiéniste, tous les deux avec le sociologue, et l'étude de la campagne, le village et la cité, et pour la poursuite de ses travaux, la discussion détaillée entre eux, en conférences amicales représentatives des différents groupes et intérêts en jeu. » 
Par sa méthode de conservative surgery qu'il réinvestit à chaque nouveau « diagnostic prospectif», les propositions de Geddes sont à chaque fois uniques dans le sens où elles résultent de la compréhension globale d'un territoire, de ses habitants et de leurs usages formant par définition un ensemble spécifique aux caractéristiques repérées.

Au-delà de l'appréhension spatiale approfondie des lieux par leur arpentage corporel et le recueil de la parole habitante lors de nombreuses rencontres, Geddes développe une attention aux temporalités et aux rythmes. Le développement urbain s'envisage alors comme un processus qui s'étend dans l'espace comme dans le temps, et dont passé, présent et futur forment un seul et même fil temporel. Le développement, généralement croissant, de la cité indienne résulte bien d'une transmission (social heritage) qui laisse à chaque fois des traces, des subsistances, ou encore des empreintes qui constituent en partie la base d'un renouveau urbain permanent. Sa démarche n'est pourtant pas celle d'un conservateur tourné vers le passé, mais bien d'un «accompagnateur» qui tente de prolonger, et non de figer, un fonctionnement des choses dans le temps tout en projetant des améliorations.

À travers l'étude plus précise du report sur la ville d'Indore (figure $4^{8}$ ), nous avons été amenés à soulever plusieurs hypothèses qui interrogent la relation entre patrimoine et projet, entre déjà-là et devenir.

\section{Mémoire du futur}

La première hypothèse invite à partir des travaux de Geddes, à réfléchir non pas sur le patrimoine, mais sur « ce qui fait patrimoine » et consisterait à dire qu'à travers la lecture d'une ville, nous pouvons lire à la fois son passé et son avenir, le passé contenant l'avenir de manière latente, inscrit dès l'origine de la cité, dans ses « gènes initiaux ». Ainsi ce devenir ferait patrimoine avant même son existence physique.

Au regard des travaux indiens de Geddes le social heritage est bien une histoire de transmission et de mémoire où, loin de suivre une démarche qui consiste à conserver au maximum les marques d'un passé plus ou moins lointain, ce qu'il décrit d'ailleurs fortement, Geddes s'inscrit dans une dynamique vitaliste, fertile et organique. Ce ne sont pas les éléments qui témoignent de l'histoire de la cité qui l'intéressent, mais bien un corpus hétérogène qui raconte une histoire, celle d'un territoire avec ses habitants, ses usages, ses pertes, ses latences et ses reconductions, des éléments transmis à travers le temps qui donnent à lire, à comprendre et à poursuivre une évolution.

8. Nous tenons à remercier pour leur aimable autorisation de reproduction la University of Strathclyde à Glasgow. 


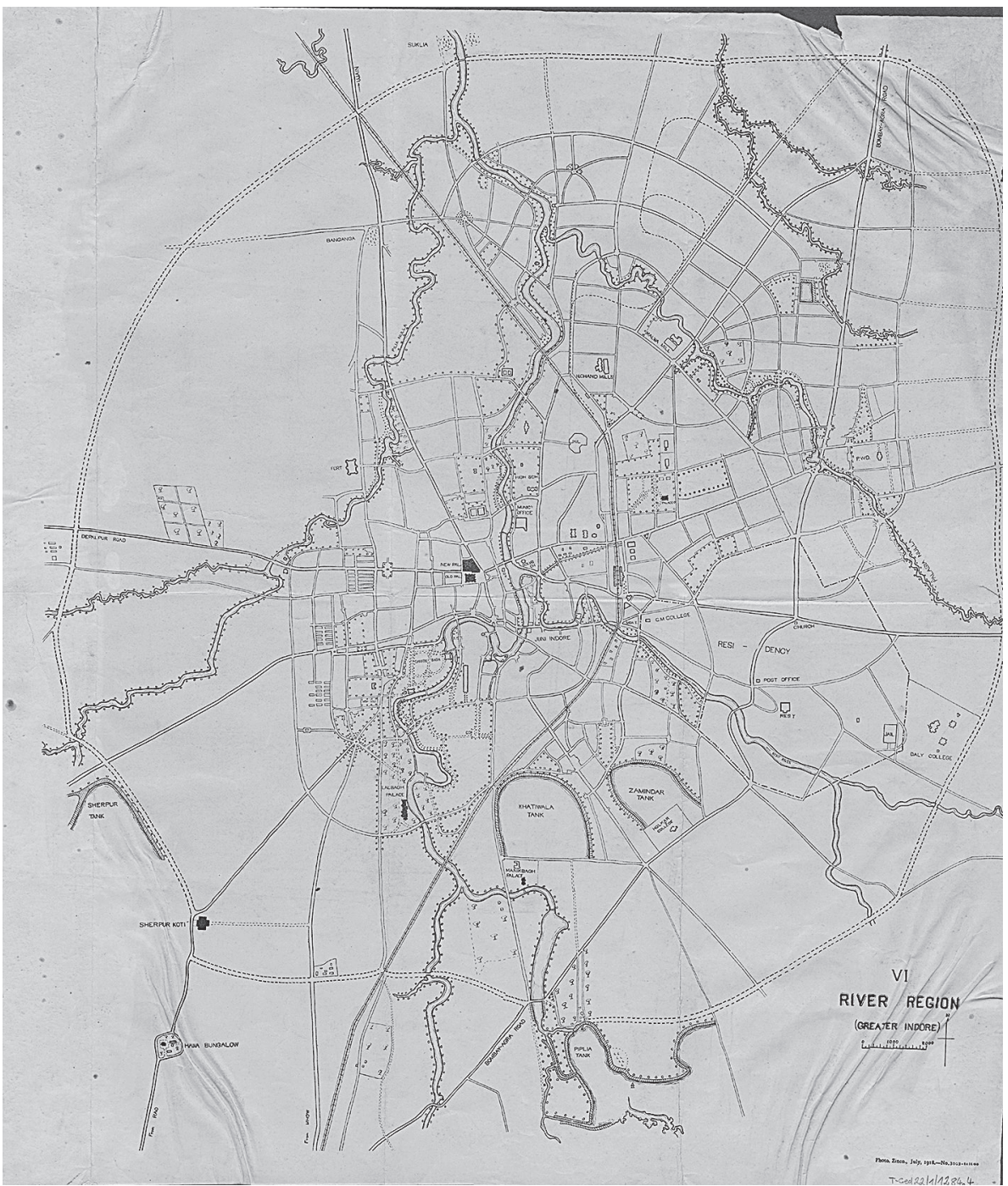

Figure 4 - VI River region (greater Indore). Carte présente dans l'édition : Geddes, P. Town Planning towards City Development. A Report to the Durban of Indore, vol. 1, Indore, Holkar State Printing Press, 1918. Collection University of Strathclyde : Patrick Geddes papers (archives reference: TGED/22/1/INDIA/1284/4).

Chaque élément du quotidien est ainsi pour Geddes susceptible de faire patrimoine comme signe, code, trace de la mémoire des lieux, s'il peut se révéler comme ayant de véritables potentialités à investir ou réinvestir dans le projet. Tangibles, intangibles, latents ou cachés, ces éléments sont repérés. Le travail du survey before planning prend ici tout son sens. Plus qu'un relevé il 
s'agit d'un véritable travail d'enquête minutieux, une expédition archéologique qui sonde la ville à tous les niveaux. Le survey geddesien est une étape essentielle qui permet de capter puis d'interpréter l'épaisseur du territoire, cette stratification temporelle qui ne cesse de se modifier et d'évoluer par l'apport successif de nouvelles strates. L'interprétation de ce palimpseste urbain construit pour Geddes un cadre et un récit pour comprendre et relire le territoire afin de pouvoir y intervenir.

Le décodage et la compréhension de ce palimpseste composent une stratigraphie urbaine originale car elle n'est pas basée sur des couches temporelles, mais sur des éléments de nature différente : le sol, le végétal, l'eau, l'air, le bâti des différentes périodes, les éléments d'ambiances relevées, les anciens usages et les actuels, la valeur symbolique des lieux et les éléments de mémoire orale que Geddes énonçait comme social heritage.

Par sa constitution variée parfois difficile à décrypter, cet ensemble s'apparente à une structure entremêlée qui s'établit dans le temps et que Geddes invite à prendre en compte dans sa globalité, comme le résultat d'une « concrescence » (d'un croître ensemble) :

«In city planning then, we must constantly keep in view the whole city, old and new alike in all its aspects and at all its levels. The transition in an Indian city, from narrow lanes and earthen dwellings to small streets, great streets and buildings of high importance and architectural beauty, form an inseparably interwoven structure. » (Geddes dans Tywhitt, 1947, p. 26) ${ }^{9}$

L'étude précise des temporalités qui composent la cité met à jour des processus qui permettent de se détacher d'une stratification uniquement temporelle pour montrer comment les strates d'époques ou de natures différentes ne se recouvrent pas les unes les autres, mais forment conjointement et dans leurs évolutions un tout, un lieu. Il s'agit alors à partir des divers éléments recueillis au cours du survey before planning de reconstituer la logique de la croissance urbaine et de la continuer pour interroger ce que Geddes énonce comme un « possible » urbain, et que nous regardons comme un « devenir ». Pour ce faire Geddes s'intéresse tout particulièrement au noyau urbain, véritable point de départ de son enquête, procédant par abduction.

«In most cities, the center and germ of origin is easily and soon discovered, as from cross-roads, from River-crossing at a Ford and later a Bridge, or from a Fair, a Palace or a Fort, as the case may be, and with characteristic varieties of

9. « Pourtant dans la planification des villes, nous devons constamment garder en vue la ville dans sa globalité, vieille et nouvelle à la fois, dans tous ses aspects et à tous ses niveaux. La transition dans une ville indienne, des passages étroits avec des habitations en terre aux petites rues, des larges rues aux bâtiments importants et à la belle architecture, tout cela forme une structure entremêlée non-séparable. » 
Bazar accordingly. Yet despite considerable experience of such enquiries, it took me a good many days of exploring and puzzling in Indore before finding its historic nucleus. »(Geddes, 1918, p. 1) ${ }^{10}$

À travers ce cœur urbain, plus qu'une lecture passéiste, ce sont les traces d'un devenir en formation qui sont perçues et décryptées. Ce futur conjugué au passé, parfois difficilement lisible, mais garant du maintien de l'équilibre vital urbain, s'apparente en tout point à une construction organique. Le « gène ancestral » contient dès sa formation l'écriture d'une évolution à venir, le tissage d'un futur en construction porté par les conditions favorables du milieu auquel il appartient.

À partir de ce parallèle, le « devenir » reste bien un « possible ». Autrement dit, une prolongation incertaine dans sa lecture, son interprétation et sa mise en œuvre dont les éléments latents originellement présents dans les couches stratifiées de la ville et de son territoire font et feront patrimoine, car étant partie prenante de ce « tout» précédemment évoqué, transmis au cours du temps.

\section{DE L'ÉPAISSEUR MÉMORIELLE AU PROJET}

La lecture et l'interprétation de ce palimpseste, qui révèlent des éléments mémoriels témoignant d'une évolution passée et à venir, constituent une étape au fondement de la planification urbaine pour Geddes. Cette étape permet de définir un cadre d'intervention en se positionnant en continuité d'un existant. Par ce constat, notre seconde hypothèse est relative à sa mise en œuvre.

La méthode de conservative surgery imaginée et mise en pratique par Geddes, serait l'articulation même permettant la prolongation tangible entre éléments de patrimoine et potentiels de projet, en intervenant comme un acte de tunning, entre préservation et actualisation de la mémoire.

Nous supposons de ce fait que la microchirurgie de Geddes est un geste qui tend à la régénération d'un milieu, rendant parfois une requalification de ses tissus urbains possible. Par des interventions locales et minutieuses, qui concrétisent des potentialités repérées en amont, c'est bien un ensemble urbain et territorial qui est visé. Il s'agit de réinvestir les marqueurs mémoriels les plus pertinents à la transmission et à l'évolution de la mémoire, dans sa matérialité et son immatérialité, en tant que potentiels de projet.

Si nous revenons maintenant au terme de conservative surgery et que nous reprenons sa traduction littérale de «chirurgie conservatrice » un paradoxe

10. «Dans la plupart des villes, le noyau, le germe original est facilement et rapidement mis au jour, à partir des carrefours, des gués qui plus tard deviennent des ponts, ou à partir d'une foire, d'un palais ou d'un fort, comme cela peut être le cas et selon les différents types de bazars. Pourtant, malgré une expérience considérable dans de telles enquêtes, il me fallut bien quelques jours d'exploration et de réflexion sur Indore avant de trouver son noyau historique. » 
s'établit entre le terme de chirurgie - qui s'apparente à une intervention qui vient modifier un état des choses - et celui de conservatrice qui inversement renvoie à la posture statique de conservation. L'inscription contraire de Geddes dans une logique biologique dite active, nous amène à interroger la différence entre l'acte même de conservation et le sens donné ici au terme de conservative.

Nous entrevoyons ainsi le sens de conservative comme permettant un maintien de la vie et l'assurance d'une transmission d'une génération à une autre, par la réparation, la modification, la reconduction ou l'ablation de tissus anciens - parfois lésés - pour en permettre le renouvellement, et non pas conserver pour ne pas " oublier ", comme une tentative de se soustraire à un processus évolutif, en cherchant à tout prix le maintien d'un même état dans la durée.

Comme le souligne Lewis Mumford dans l'introduction de Patrick Geddes in India, Geddes s'inscrit dans le respect et la compréhension d'un ordre naturel qui fait la particularité de sa démarche : «He saw both cities and human being as wholes; and he saw the processes of repair, renewal and rebirth as natural phenomena of development » (Mumford dans Tyrwhitt, 1947, p. 11) ${ }^{11}$.

Plus judicieusement il nous semble que conservative pourrait alors se traduire par réparatrice plutôt que conservatrice, d'autant que son association au terme surgery, induit une gestuelle réparatrice, voire salvatrice, résonnant plutôt comme une marque de projet, un acte de visibilité et de concrétisation.

La pratique de conservative surgery, par sa réinterprétation des éléments qui font patrimoine en tant que potentiels de projet et par ses interventions significatives locales, engendre un renouveau urbain (comme c'est le cas avec le réseau discret formé, les temples qu'il met en évidence ou encore le repérage systématique des arbres en regard d'espaces potentiellement récupérables). En établissant une réciprocité permanente entre les différents niveaux d'études, cette pratique urbaine marque nettement la volonté de Geddes de respecter un équilibre naturel, en excluant toute posture de table rase - n'entrevoyant que l'avenir - et à l'opposé de la conservation totale - tournée vers le passé - qui empêcherait tout renouvellement.

Le passé, dans l'introduction de Lewis Mumford, correspondrait à de vieilles racines permettant la croissance et le développement de jeunes pousses, constituantes d'une « surépaisseur » amenée à se stratifier à son tour avec ses pertes, ses traces et ses reconductions à la manière d'un cycle naturel : «Just because Geddes respected the old roots of regional culture, he had no interest in limiting its expression to some historic moment of the past : if the roots were

11. «Il concevait la cité et ses habitants comme un tout, et les processus de réparation, réhabilitation et renaissance urbaines comme des phénomènes naturels. » 
alive, they would keep on putting forth new shoots, and it was in these new shoots that he was interested» (Mumford dans Tyrwhitt, 1947, p. 8) ${ }^{12}$.

\section{MÉMOIRE NATURELLE ET AMBIANCE URBAINE}

Geddes n'oubliait jamais que toute ville est construite sur un territoire préanthropique qui la façonne. Mettre en valeur cette mémoire naturelle fait partie de son travail et constitue pour lui un élément caractéristique et toujours singulier des ambiances urbaines. En cela, les travaux indiens de Geddes anticipent en quelque sorte ceux qui viendront bien plus tard sur les ambiances urbaines. Geddes regarde la ville comme un écosystème urbain, prenant en compte des aspects comme la réverbération thermique, les flux d'air et leurs dynamiques en relation avec les surfaces ombragées, les spécificités de la faune et de la flore urbaines, la fertilité et l'hydrologie des sols, l'insalubrité ou la qualité de l'eau urbaine, etc. Ses petites interventions déjà décrites de conservative surgery mettent en avant la présence d'un arbre de grande taille auprès de chaque temple et l'importance de son ombre dans ce climat chaud. Il propose dans ce tissu dense, de créer de petites ouvertures, pour faire un peu de place et « faire place », d'y planter d'autres arbres, d'y ajouter une fontaine d'eau publique. Il pense la végétalisation urbaine avec des arbres fruitiers pour participer à l'autonomisation alimentaire des habitants de la cité... (figure $5^{13}$ ).

En ce qui concerne l'aspect botanique, Geddes s'intéresse aux paramètres qui influent directement sur l'ambiance urbaine, comme le montre ce texte (Lucknow) lorsqu'il parle des friches urbaines et de la viabilité des projets de plantation d'arbres dans celles-ci. Il affirme :

"The cost of tree planting is a frequent objection. This can be mitigated by the establishment of municipal tree nursery on one of many available sites of good cultivated, or cultivatable, land that are at present unoccupied. [...] Everyone appreciates shade, but few realise how great a secondary service is performed by a tree in absorbing not only the radiant heat and light from the sun but also from the buildings and road surfaces all around it. Trees grow dusty, but dustcatching is also useful. Better dust upon the leaves of the trees than in the lungs of the citizens. [...] The judicious planting of trees may also increase breezes instead of impeding them. » (Geddes, 1917c, p. 67) ${ }^{14}$

12. « C'est justement parce que Geddes respectait les plus vieilles racines des cultures régionales qu'il n'avait aucun intérêt à rattacher ses réflexions historiques : si les racines vivaient encore, elles devaient continuer d'exister au travers de nouvelles pousses, et c'est ces nouvelles pousses qui intéressaient Geddes. »

13. Nous tenons à remercier pour leur aimable autorisation de reproduction les éditions Lund Humphries à Londres.

14. « Le coût des plantations d'arbres est une objection fréquente. Ceci pourrait être nuancé par l'implantation de pépinières municipales dans certaines des très nombreuses friches disponibles. 


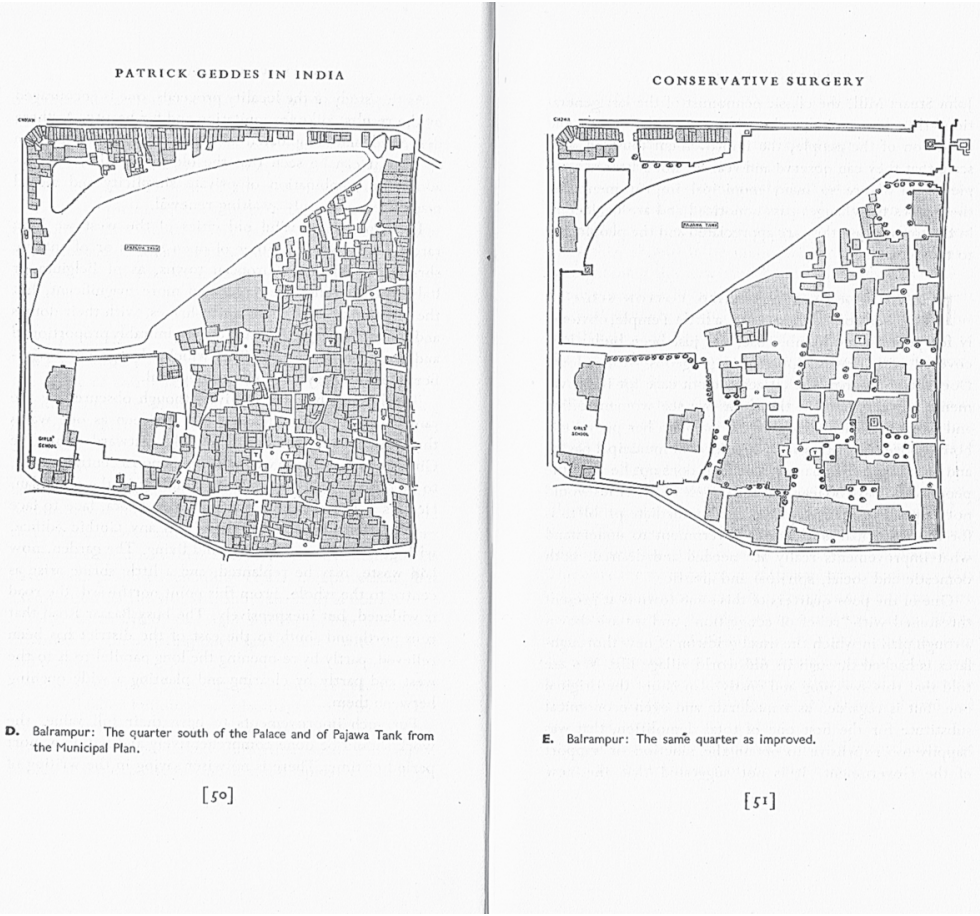

Figure 5 - Conservative Surgery in Balrampur présente dans l'édition : Geddes, P. Patrick Geddes in India, édité par J. Tyrwhitt, H. V. Lanchester et A. Geddes, introduction de Lewis Mumford, Londres, Lund Humphries, 1947.

Il est aussi très intéressant de voir comment Geddes valorise les éléments hydrauliques présents dans le territoire préanthropique sous-jacent à la ville. Une sorte de mémoire naturelle, qui est lisible par les tanks, les réservoirs des villes indiennes. Contrairement à l'opinion négative de la municipalité envers ces réservoirs d'eau qui, selon elle, favorisent la prolifération de moustiques et en conséquence, des maladies, Geddes propose de les conserver, de les nettoyer fréquemment et de réinstaller des espèces animales pour recréer un écosystème et ainsi, contrôler la population de moustiques :

[...] Tout le monde apprécie l'ombre, et les arbres n'absorbent pas seulement la radiation solaire et la chaleur du sol, mais aussi celle des bâtiments et surfaces adjacentes. Les arbres poussent dans la poussière et la captent, ce qui est aussi très utile. Il est préférable que la poussière soit sur les feuilles que dans les poumons des citoyens. [...] Une plantation judicieuse d'arbres devrait également augmenter les brises au lieu de les empêcher. » 
«The many values of the village reservoirs or tanks are seldom appreciated by sanitarians or those in administrative positions. Much could be said of their value during the long dry season in maintaining the water level both in wells and in the soil. A town rich in these reservoirs, and sloping towards them, is assured against flooding by rain-bursts. Sudden floods often overflow insufficient drains and storm water channels and destroy properties in lower areas of towns where storage tanks, such as would have been constructed by old-fashioned Indian engineers, would have prevented inundation. The cooling value of reservoirs has an appreciable influence on health and comfort, often forgotten by those who live in civil stations outside the town, with lofty rooms, shady verandahs and spacious gardens. [...] flows through the streets, especially if these are shaded by trees, and appreciably reduces the temperature of the area. " (Geddes, 1917d, p. 36) ${ }^{15}$

Ce travail colossal de réactivation et de prolongation mémorielle par la pratique du territoire dans ses divers aspects amène une dernière hypothèse qui interroge le rôle de la représentation, de l'interprétation et de la transmission de ces données.

\section{LA MARCHE, LA CARTE ET LE RÉCIT : À LA CROISÉE DES MÉMOIRES}

À travers les divers documents produits par Geddes lors de ses séjours en Inde, nous supposons en effet que la représentation graphique - ici plus particulièrement la carte - et la narration écrite ne peuvent être saisies que par la lecture de l'une à travers l'autre, comme deux objets indissociables. Par cette complémentarité semble se former le support de projection d'un passé et d'un devenir, dans lequel se lit la mémoire des lieux.

Car si carte et récit semblent au premier abord s'appréhender comme deux éléments de retranscription à part entière, ils sont, nous semble-t-il, pour Geddes, un seul et même outil projectuel. Très nettement lorsque nous superposons carte et récit nous retrouvons les sensations d'un voyage.

Ainsi, nous pénétrons à notre tour les couches stratifiées d'un palimpseste urbain que nous ne connaissons qu'à travers les documents, en naviguant entre passé, présent et avenir, mais également entre les rues étroites d'Indore.

15. «Il y a de nombreuses raisons qui font que ces réservoirs d'eau ou tanks sont très peu appréciés par l'administration locale. Beaucoup de choses pourraient être dites en leur faveur, spécialement pendant la saison sèche, en garantissant le niveau d'eau des puits et celui de la nappe phréatique. Une ville riche de ces réservoirs procure une protection contre les inondations. Souvent soudaines, celles-ci dépassent les capacités de drainage des canaux d'irrigation et détruisent des propriétés sur les zones les plus basses des villages, alors que les réservoirs de stockage d'eau comme ceux que les vieux ingénieurs indiens ont construits auraient pu les prévenir. La valeur de rafraîchissement de ces réservoirs a une influence appréciable sur la santé et le confort, souvent oubliés par ceux qui habitent dans les stations civiles, à l'extérieur de la ville, avec d'amples chambres, porches à l'ombre et jardins spacieux. [...] La différence de température de l'air du réservoir provoque une circulation d'air, en particulier si les rues sont à l'ombre des arbres, ce qui réduit sensiblement la température de la zone. » 
«Thus, for instance, after admiring this view north-east - architecturally one of the best in Indore - let the observer turn up-stream, and note how fine would be the effect of a new Temple placed upon the round bastion of the old Rampart, and built to command this Ford, upon the main River, (now the Causeway to Ara Bazar). For here a white temple with graceful spire would be seen from far and near, and doubled by its reflection mirrored in the stream. » (Geddes, 1918, p. 8) ${ }^{16}$

La force projectuelle constituée par l'association de ces deux formes de narration réside en majeure partie dans la précision graphique et écrite, qui rend compte pas à pas d'une lecture mémorielle et historique. Chacun de ces deux supports devient une retranscription labyrinthique où s'enchevêtre une lecture partagée du temps, de l'espace et de la société.

En amont de cette narration, le survey before planning convoque par la pratique de la marche, de la rencontre et de la projection une multiplicité de mémoires, qui convergent à travers l'arpenteur. Qu'il s'agisse d'une mémoire humaine, personnelle, ou encore naturelle, cet ensemble constitue le socle d'un devenir en construction.

Ainsi cette «mémoire sociale » se dédouble en étant en partie propre à chacun et partagée par une communauté. Par sa sollicitation elle permet de faire de chaque habitant, voyageur, ou encore ingénieur des acteurs de leur propre territoire en les envisageant à juste titre comme indissociables du système urbain auquel ils prennent part. Car au-delà de ses considérations biologiques et écosystémiques, Geddes tendait plus que tout à la valorisation des individus et de leurs savoir-faire locaux, quels que soient leur statut et leur place dans la société. De cette manière, lors des surveys before planning, Geddes s'entourait d'équipes locales capables de l'accompagner dans la compréhension et la retranscription du territoire qu'il arpentait. Nous savons d'ailleurs que les différents redessins présents dans le report d'Indore s'appuient sur des cartes existantes produites précédemment par les institutions indiennes.

L'interaction mémorielle, qui mêle à la fois données sensorielles, personnelles, communes ou encore naturelles, est précisément ce qui fait naître un récit des lieux appelant encore et toujours à son prolongement et à sa relecture. Par la carte il s'agit alors de raconter une histoire de prime abord spatiale dont les diverses formes, fonctions et mouvements prennent vie à travers un récit écrit. Par la narration écrite, qui inclut et fixe sur le papier un recueil oral, il

16. «Ainsi, par exemple, après avoir admiré cette vue nord-est - architecturalement parlant l'une des plus belles à Indore - nous laissons l'observateur se retourner, et imaginer le bel effet que produirait un nouveau temple installé sur le bastion arrondi du vieux rempart, construit pour surveiller et maîtriser le gué au-dessus la rivière principale (désormais devenu la chaussée qui conduit à l'Ara Bazar). Ici, un temple blanc aux majestueuses flèches serait remarqué de près comme de loin, et prolongé par son reflet dans le miroir du courant d'eau. » 
s'agit de nous orienter dans l'espace cartographique et de relater une évolution dans le temps en rendant possible une projection passée, présente et à venir. L'association de ces deux médias invite à un véritable arpentage mental, un espace de projection, qui en suivant le parcours d'une expédition in situ donne à lire une « mémoire des lieux » qui loin d'être figée sur le papier appelle à se renouveler par la prolongation de sa transmission.

La tendance actuelle qui consiste à faire projet par l'apport collectif, sousentendu en s'appuyant principalement sur une ressource citoyenne considérée comme actrice principale du renouvellement de son territoire, fait écho à la démarche prônée et appliquée par Patrick Geddes il y a de cela plus d'un siècle. En réintégrant l'histoire urbaine, la mémoire naturelle, la parole habitante, l'héritage culturel ou encore l'inconscient collectif dans nos propositions de planification urbaine, il s'agit de produire un récit nouveau et d'orienter le devenir urbain. Cela ne signifie pas s'opposer à toute construction nouvelle ni à l'idée de projet urbain, bien au contraire, c'est en travaillant ces « variables » que le « nouveau » dans la ville trouve un profond enracinement culturel grâce au travail de mémoire et à la préservation des valeurs qui lient une société à un lieu. Mémoire et avant-garde se côtoient pour réactiver à travers des usages l'expérience urbaine. Explorer les limites entre urbain et naturel, ville construite et ville vécue, en vue de construire une théorie-pratique de la planification et de l'intervention urbaines.

Le travail sur la mise en place de micro-initiatives locales variées, notamment en lien avec la nature, et la prise en compte d'un «déjà-là » dans ses divers aspects, tel un ensemble mémoriel à définir et à possiblement réinvestir dans le projet, fait de Geddes un auteur étonnamment pertinent pour notre contemporanéité.

\section{RÉFÉRENCES BIBLIOGRAPHIQUES}

Ferraro G. 1998. Rieducazione Alla Speranza. Patrick Geddes Planner in India (1914-1924), Milan, Jaca Book.

Geddes, P. 1994 [1884]. "An Analisis of the Principles of Economics », dans J Martínez Alier (sous la dir. de), Los Principios de la Economía Ecológica, Madrid, Fundación Argentaria.

Geddes, P. 1905. « Civics as Applied Sociology », Sociological Papers, vol. 1 et 2. Geddes, P. 1915. Reports on the Town in the Madras Presidency Visited by Professor Geddes 1914-1915, Madras, Government Press.

Geddes, P. 1917a. Town planning in Lahore. A report to the Municipal Council, Lahore, Municipal Press.

Geddes, P. 1917b, Town planning in Nagpur. A report to the Municipal Committee, Nagpur, Municipal Press.

Geddes, P. 1917c. Town Planning in Lucknow. A Second Report to the Municipal Council, Lucknow, Murray's London Printing Press. 
Geddes, P. 1917d. Town Planning in Balrampur. A Report to the Honourable the Maharaja Bahadur, Lucknow, Murray's London Printing Press.

Geddes, P. 1918. Town Planning towards City Development. A Report to the Durban of Indore, Indore, Holkar State Printing Press, vol. 1 et vol. 2.

GedDEs, P. 1919. « The Temple Cities », Modern Review, vol. 25, n 3.

Geddes, P. 1915. Cities in Evolution, London, William and Norgate.

Oyón, J. L. 2011. « Dispersión Frente a Compacidad. La Paradoja del Urbanismo Protoecológico », Ciudad y Territorio. Estudios territoriales, $\mathrm{n}^{\circ}$ 169-170, p. 515-532.

TyrwhitT J. (sous la dir. de) 1947. Patrick Geddes in India, Londres, Lund Humphries. 